\title{
Syntheses and reactions of methyl [3-(4-phenyl-thiazol-2-yl)- thioureido] alkanoates and related compounds
}

\author{
Walid Fathalla \\ Department of Mathematical and Physical Sciences, Faculty of Engineering, \\ Suez Canal University, Port Said, Egypt \\ E-mail:walid399@yahoo.com
}

\section{Dedicated to Prof. Ibrahim A. I. Aly for his efforts in Organic Chemistry at Suez Canal University}

\begin{abstract}
Thiazole thioureas 4a-d and $\mathbf{6}$ bearing an amino acid ester residue were prepared by a one-pot sequential reaction of methyl thiocarbamate 2 with amino acid methyl ester hydrochlorides. Some chemoselective reactions of $\mathbf{4 a , b}$ with alkyl halides were studied.
\end{abstract}

Keywords: Non-nucleoside RT inhibitors, PETT, thiazole thioureas, amino acids, isothioureas, thiazolidin-4-one, intermolecular hydrogen bond, intramolecular hydrogen bond interactions

\section{Introduction}

Acquired immune compromised deficiency syndrome (AIDS) is caused by HIV (a retrovirus) affecting 33 million people worldwide, mostly affecting women and children in Sub-Sahara Africa. ${ }^{1}$ Three million people around the world die of AIDS each year and, so far, more than 25 million people have died of the disease.

Lately, multiple antiretroviral agents have been produced to block replication of the HIV-1 virus by blocking HIV reverse transcriptase ${ }^{2}$ or by blocking HIV protease. ${ }^{3}$ Among the most important anti-retroviral agents recently introduced are the non-nucleoside reverse transcriptase inhibitors (NNRTI), such as nevirapine ${ }^{4}$ and delavirdine ${ }^{5}$ able to reduce RT inhibition to subnanomolar concentrations.

Several heterocyclic thioureas have been reported as a new class of potent NNRTIs such phenethylthiazolyl-thiourea (PETT) derivatives. ${ }^{6-9}$ Uckun et al. ${ }^{10}$ described the synthesis of a series of thiazole thioureas with alkyl, aryl, heteroaryl substituents as newly identified NNRTI of HIV, including mutant strains of HIV, and effective in the treatment of multi-drug resistant HIV infection. 
We have recently reported a new and efficient synthesis of novel quinazoline thioureas bearing an amino acid ester residue based on domino reaction of $N$-(2-cyanophenyl)benzimidoyl isothioyanate with amino acid methyl ester hydrochlorides. ${ }^{11}$

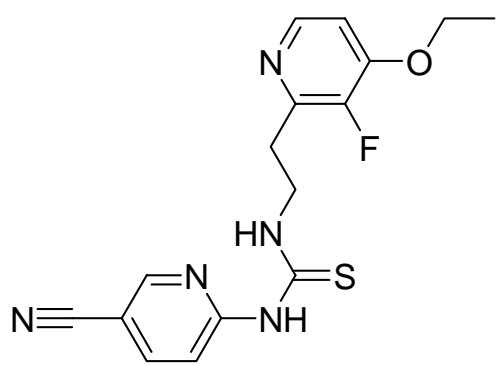

PETT1

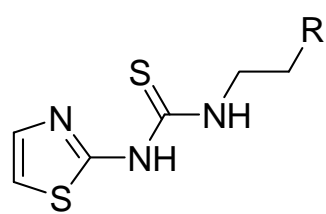

thiazole thiourea<smiles>[R]C(=NC(=S)Nc1nc(-c2ccccc2)nc2ccccc12)C(=O)OC</smiles>

quinazoline thiourea

Figure 1

\section{Results and Discussion}

The synthesis of biologically active amino acid coupled derivatives was considered to be of a major interest. ${ }^{11-14}$ In this paper, we described the development of a new series of thiazole thioureas bearing amino acid esters which can be used as potent NNRTIs.

The required thiazole thioureas 4a-d and $\mathbf{6}$ were easily prepared starting from 4-phenylthiazol-2-ylamine (1) through methyl thiocarbamate $\mathbf{2}$ as a key intermediate in one-pot sequential reaction strategy (Scheme 1). First step; 4-phenyl-thiazol-2-ylamine (1) reacts with equimolar amount of carbon disulfide in basic media $(\mathrm{KOH})$ using DMSO as solvent at $80^{\circ} \mathrm{C}$ for $2 \mathrm{~h}$. The reaction mixture was then cooled to $0{ }^{\circ} \mathrm{C}$ and dimethyl sulfate was added simultaneously. The in situ generated methyl thiocarbamate $\mathbf{2}$ was extracted and used without further purification.

Compound $\mathbf{2}$ is an interesting reactive intermediate for the synthesis of a variety of heterocyclic compounds. ${ }^{15}$ This reactivity is due to leaving group ability of SMe group when reacting with nucleophiles. Thus, equimolar amount of amino acid ester hydrochloride 3 adds to in situ generated methyl thiocarbamate 2 in the presence of triethylamine in ethyl alcohol to afford methyl [3-(4-phenyl-thiazol-2-yl)-thioureido] alkanoate 4. Similarly, methyl paminobenzoate 5 adds to 2 to afford thiourea 6 .

The syntheses of 4a-d and 6 reported herein have the advantage of one-pot synthesis in addition to operational simplicity and availability giving a series of very interesting compounds. 


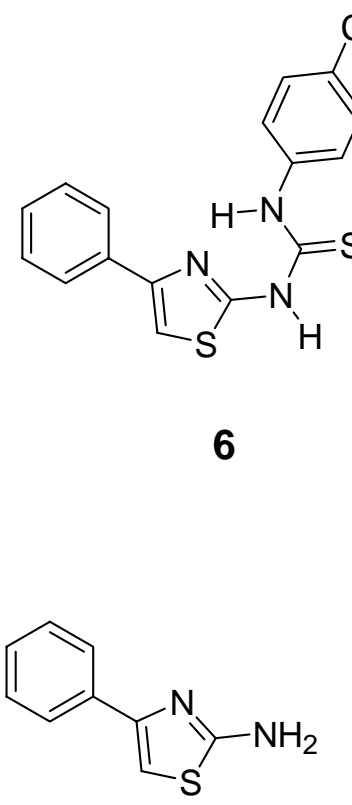

1<smiles>S=C(NCc1ccccc1)Nc1nc(-c2ccccc2)cs1</smiles>

$\left\{\begin{array}{l}7 \\ \begin{array}{c}\text { EtOH, } \mathrm{Et}_{3} \mathrm{~N}, 70^{\circ} \mathrm{C}, 4 \mathrm{~h} \\ \text { benzyl amine }\end{array}\end{array}\right.$

1. $\mathrm{CS}_{2}, \mathrm{DMF}, \mathrm{KOH}, 25^{\circ} \mathrm{C}$

2. dimethyl sulfate

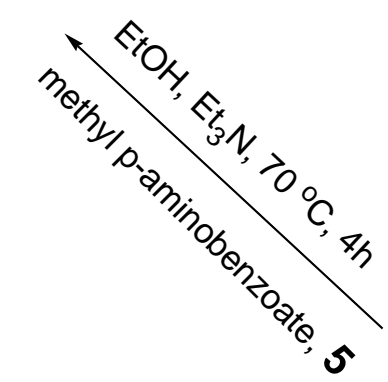

[<smiles>CSC(=S)N=c1[nH]c(-c2ccccc2)cs1</smiles>

\section{$\mathrm{NH}_{2}\left(\mathrm{CH}_{2}\right)_{n} \mathrm{CHRCOOCH}_{3} . \mathrm{HCl}, 3$ \\ $\mathrm{EtOH}, \mathrm{Et}_{3} \mathrm{~N}, 70^{\circ} \mathrm{C}, 4 \mathrm{~h}$}

\begin{tabular}{l|ll}
$\mathbf{3 , 4}$ & $\mathrm{n}$ & $\mathrm{R}$ \\
\hline $\mathbf{a}$ & 0 & $\mathrm{H}$ \\
$\mathbf{b}$ & 1 & $\mathrm{H}$ \\
$\mathbf{c}$ & 0 & $\mathrm{CH}_{3}$ \\
d & 0 & $\mathrm{CHCH}_{2}\left(\mathrm{CH}_{3}\right)_{2}$
\end{tabular}

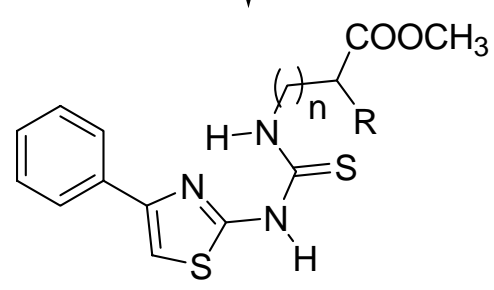

4

\section{Scheme 1}

The structure assignments of amino acid esters 4a-d and $\mathbf{6}$ are based on spectral and physicochemical analysis. All isolated products exhibited a rather interesting conformation as indicated from all ${ }^{1} \mathrm{H}$ NMR spectra, figure 2. Thus, the ${ }^{1} \mathrm{H}$ NMR spectrum of $\mathbf{4 b}$ showed three signals; a quartet, a triplet and a singlet at $\delta 4.12,2.83$ and $3.69 \mathrm{ppm}$ consequent to two $\mathrm{CH}_{2}$ groups of the $\beta$-alanine residue and $\mathrm{OCH}_{3}$ group, respectively.

In addition the ${ }^{1} \mathrm{H}$ NMR spectrum of $\mathbf{4 b}$ gave two exchangeable singlets at $\delta 11.29$ and 10.57 ppm corresponding to two $\mathrm{NH}$ groups. This implies that $\mathrm{N} 3-\mathrm{H}$ group participate in a strong intramolecular hydrogen bond interaction with the thiazole nitrogen of the type N3$\mathrm{H} \cdots \mathrm{N}=\mathrm{C} .{ }^{10,11,16}$ The other $\mathrm{N} 1-\mathrm{H}$ group is involved in an intermolecular hydrogen bond with 
either the thiocarbonyl of the thiourea of the type $\mathrm{N} 1-\mathrm{H} \cdots \mathrm{S}=\mathrm{C},{ }^{17-20}$ or the carbonyl of the amino acid residue of the type $\mathrm{N} 1-\mathrm{H} \cdots \mathrm{O}=\mathrm{C} .^{21-23}$

The intermolecular hydrogen bond could be distinguished by comparison of the ${ }^{1} \mathrm{H}$ NMR spectra with similar compound lacking the ester function group. 1-Benzyl-3-(4-phenyl-thiazol-2yl)-thiourea 7 was similarly prepared by the reaction of benzyl amine with insitu generated methyl thiocarbamate 2, scheme 1. The ${ }^{1} \mathrm{H}$ NMR spectrum of 7 shows chemical shift at 11.49 ppm due to NH group participating in an intramolecular hydrogen bond; while gave chemical shift at $6.38 \mathrm{ppm}$ due to $\mathrm{N} 1 \mathrm{H}$ group.

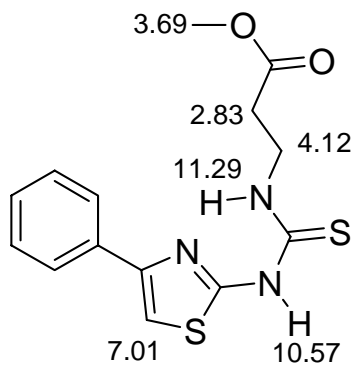

4b

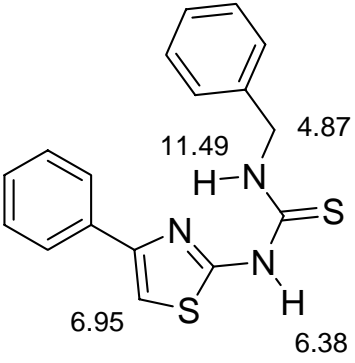

7

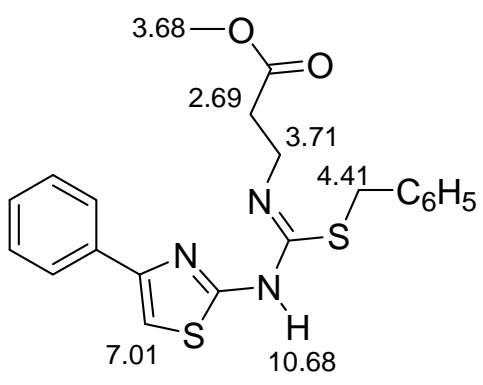

10b

Figure 2. Selected ${ }^{1} \mathrm{H}$ NMR data of compounds $\mathbf{4 b}, \mathbf{7}$ and $\mathbf{1 0 b}$.

The reaction of glycine derivatives 4a with absolute ethanol in the presence of potassium hydroxide furnished the trans-esterification product 8 (Scheme 2). The reaction of methyl [3-(4phenyl-thiazol-2-yl)-thioureido] alkanoates 4a,b (Gly and $\beta$-Ala) with electrophiles might give more evidence for structure elucidation beside chemical and biological importance. Thus, the reaction of 4a,b with allyl bromide and benzyl bromide in the presence of $\mathrm{Et}_{3} \mathrm{~N}$ gave a single tautomeric chemoselective $S$-alkylated derivatives $\mathbf{9 a , b}$ and $\mathbf{1 0 a}, \mathbf{b}^{24-26}$ induced by hydrogen bond interactions. Earlier we reported the chemoselective $S$-alkylation reaction in similar compounds afforded only a single compound preserving the intramolecular hydrogen bond. ${ }^{11}$

The ${ }^{1} \mathrm{H}$ NMR spectrum clearly deduced the alkylation site and the position of double bond. The ${ }^{1} \mathrm{H}$ NMR spectrum of $\mathbf{1 0 b}$ has shown a broad singlet at $\delta 10.68$ attributed to N1H group partipating in an intermolecular hydrogen bond of the type $\mathrm{N} 1-\mathrm{H} \cdots \mathrm{O}=\mathrm{C},{ }^{21-23}$ figure 2 . Also, the ${ }^{1} \mathrm{H}$ NMR spectrum of 10b gave three signals at $\delta 4.41,3.71$ and $2.69 \mathrm{ppm}$ consequent to $\mathrm{SCH}_{2}{ }^{24}$ and two $\mathrm{CH}_{2}$ groups of the $\beta$-alanine residue, respectively.

Thiazolidin-4-one derivatives are known to exhibit diverse bioactivities such as antiHIV. ${ }^{27}$ Hence, it seems of interest to attach thiazolidin-4-one bearing amino acid ester residue to amino thiazole moieties in single molecular framework to enhance the biological activity.

The reaction of methyl [3-(4-phenyl-thiazol-2-yl)-thioureido] alkanoates 4a,b (Gly and $\beta$ Ala) with methyl chloroacetate resulted in the formation of thiazolidines 11a,b. The ${ }^{1} \mathrm{H}$ NMR spectrum of 11a shows three singlets at $\delta 4.64,3.93$ and 3.78 ppm typically associated with an $\mathrm{NCH}_{2}, \mathrm{SCH}_{2} \mathrm{CO}$ and $\mathrm{OCH}_{3}$, respectively. This structure was in good agreement with our 
previous results with methyl [4-oxo-2-(2-phenylquinazolin-4-ylimino)thiazolidin-3-yl]acetate preparation, figure $3 .{ }^{11}$

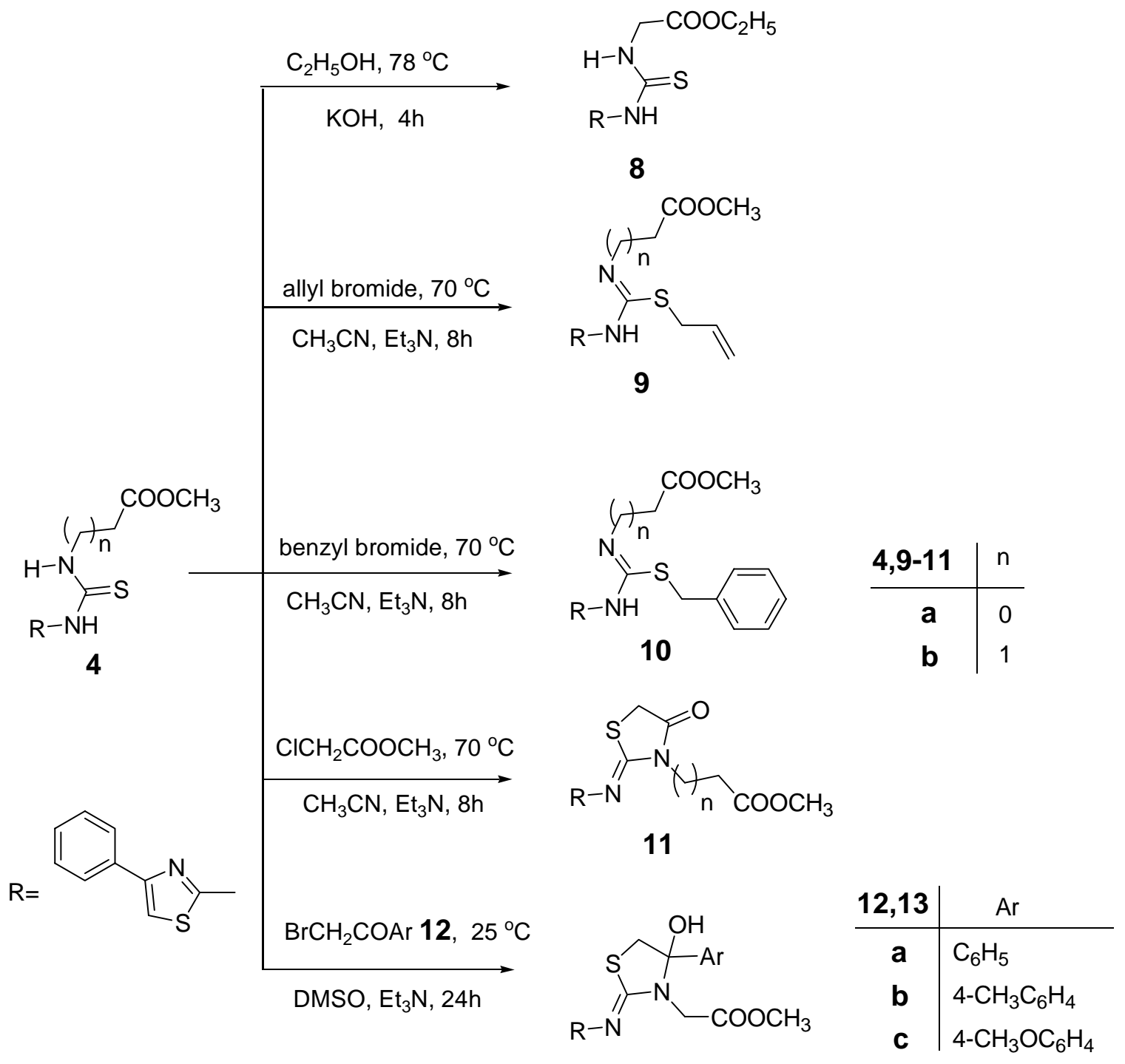

13

\section{Scheme 2}

Similarly, the reaction of glycine derivative 4a with phenacyl bromides 12 afforded 4hydroxythiazolidines 13a-c. The ${ }^{1} \mathrm{H}$ NMR spectra of 4-hydroxythiazolidines 13a-c gave completely different pattern compared to thiazolidine 11a,b. The ${ }^{1} \mathrm{H}$ NMR spectrum of $\mathbf{1 3 b}$ shows two doublets centered at $\delta 4.40$ and $\delta 3.63 \mathrm{ppm}\left(J_{\mathrm{AB}}=17.2 \mathrm{~Hz}\right)$ corresponding to an $\mathrm{AB}$ system of the prochiral hydrogen atoms of the $\mathrm{NCH}_{2}$ group. In addition, apparent two doublets are displayed at $\delta 3.57$ and $3.48 \mathrm{ppm}\left(J_{\mathrm{AB}}=11.8 \mathrm{~Hz}\right)$ resulting from $\mathrm{A}$ and $\mathrm{B}$ parts of the $\mathrm{AB}$ quartets of $\mathrm{SCH}_{2}$ groups. ${ }^{11}$ 


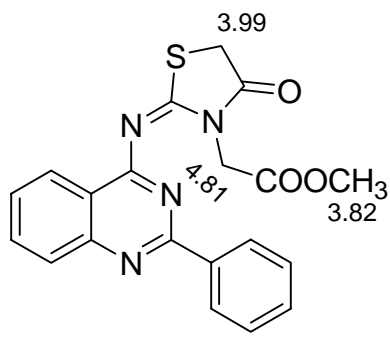

quinazoline thiazolidine

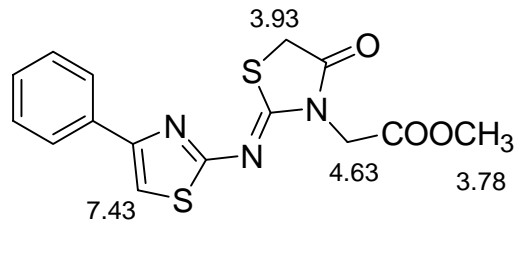

$11 \mathrm{a}$

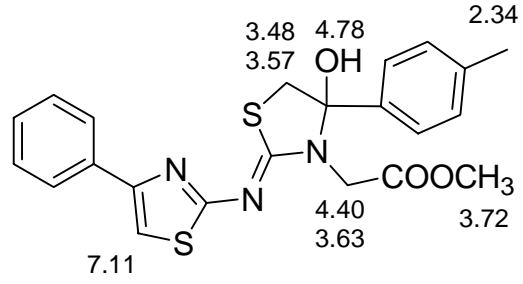

$13 b$

Figure 3. Selected ${ }^{1} \mathrm{H}$ NMR data of compounds 11a, 13b and quinazoline thiazolidine. ${ }^{11}$

\section{Experimental Section}

General Procedures. Solvent were purified and dried in the usual way. The boiling range of the petroleum ether used was $40-60{ }^{\circ} \mathrm{C}$. Thin layer chromatography (TLC): silica gel $60 \mathrm{~F}_{254}$ plastic plates (E. Merck, layer thickness $0.2 \mathrm{~mm}$ ) detected by UV absorption.

Elemental analyses were performed on a Flash EA-1112 instrument at the Microanalytical laboratory, Faculty of Science, Suez Canal University, Ismailia, Egypt. Melting points were determined on a Buchi 510 melting-point apparatus and the values are uncorrected. NMR spectra measured with Bruker $(200 \mathrm{MHz})$ and TMS $(0.00 \mathrm{ppm})$ was used as internal standard. The starting compounds 1 was prepared according to described methods. ${ }^{28}$

\section{General procedure for preparation of 4, 6 and 7}

To a vigorously stirred solution of 4-phenyl-thiazol-2-ylamine (1) (0.9 g, $5.0 \mathrm{mmol})$ in dimethylsulfoxide $(10 \mathrm{ml})$ at room temperature, carbon disulfide $(0.4 \mathrm{ml}, 6.5 \mathrm{mmol})$ and potassium hydroxide $(0.31 \mathrm{~g}, 5.5 \mathrm{mmol})$ solution in $2 \mathrm{~mL} \mathrm{H}_{2} \mathrm{O}$ were added dropwise simultaneously over $30 \mathrm{~min}$, the mixture was then allowed to stir for $30 \mathrm{~min}$ in water bath at 80 ${ }^{\circ} \mathrm{C}$. The reaction mixture was cooled in an ice bath to $0{ }^{\circ} \mathrm{C}$ and dimethyl sulfate $(0.5 \mathrm{~mL}, 5.0$ mmol) was added dropwise for 15 min under cooling. Stirring was continued for $3 \mathrm{~h}$, the reaction mixture was poured into ice-water and then it was extracted with chloroform. The solvent was removed by distillation under reduced pressure. Thus, the obtained crude methyl thiocarbamate 2 dissolved in ethanol $30 \mathrm{~mL}$ cooled to $10{ }^{\circ} \mathrm{C}$ and was treated with nucleophiles [amino acid methyl ester hydrochloride $3(5.0 \mathrm{mmol})$ and triethyl amine $(0.7 \mathrm{~mL}, 5.0 \mathrm{mmol})$; methyl $p$ aminobenzoate $5(0.75 \mathrm{~g}, 5.0 \mathrm{mmol})$ or benzyl amine $(0.55 \mathrm{~mL}, 5.0 \mathrm{mmol})]$. Stirring was continued for $3 \mathrm{~h}$ at $70{ }^{\circ} \mathrm{C}$. The reaction mixture was concentrated and the solid obtained was filtered, washed with water, dried and recrystallized from ethanol.

Methyl [3-(4-phenyl-thiazol-2-yl)-thioureido] acetate (4a). From $\mathrm{GlyOCH}_{3} \cdot \mathrm{HCl}$ 3a $(0.65 \mathrm{~g})$. Colorless crystals (1.05 g, $67 \%$ ); $\mathrm{mp} 212-213{ }^{\circ} \mathrm{C} .{ }^{1} \mathrm{H}$ NMR $\left(200 \mathrm{MHz}, \mathrm{CDCl}_{3}\right): \delta 11.51(1 \mathrm{H}, \mathrm{bs}$, $\mathrm{D}_{2} \mathrm{O}$ exchangeable, $\left.\mathrm{NH}\right), 9.77\left(1 \mathrm{H}, \mathrm{bs}, \mathrm{D}_{2} \mathrm{O}\right.$ exchangeable, $\left.\mathrm{NH}\right), 7.92(2 \mathrm{H}, \mathrm{d}, J=8.0 \mathrm{~Hz}, \mathrm{ArH})$, 7.48-7.32 (3H, m, ArH), $7.03\left(1 \mathrm{H}, \mathrm{s}, \mathrm{CH}\right.$-thiazole), $4.57\left(2 \mathrm{H}, \mathrm{d}, J=4.2 \mathrm{~Hz}, \mathrm{NHCH}_{2}\right), 3.86(3 \mathrm{H}$, 
s, $\mathrm{OCH}_{3}$ ). Anal. Calcd. For $\mathrm{C}_{13} \mathrm{H}_{13} \mathrm{~N}_{3} \mathrm{O}_{2} \mathrm{~S}_{2}$ (307.4): C, 50.79; H, 4.26; N, 13.67; Found: C, 50.63; H, 4.19; N, 13.61.

Methyl 3-[3-(4-phenyl-thiazol-2-yl)-thioureido] propanoate (4b). From $\beta-\mathrm{AlaOCH}_{3} \cdot \mathrm{HCl} \mathbf{3 b}$ (0.7 g). Colorless crystals (1.35 g, $85 \%$ ); mp 202-203 ${ }^{\circ} \mathrm{C} .{ }^{1} \mathrm{H}$ NMR $\left(200 \mathrm{MHz}, \mathrm{CDCl}_{3}\right): \delta 11.29$ $\left(1 \mathrm{H}, \mathrm{bs}, \mathrm{D}_{2} \mathrm{O}\right.$ exchangeable, $\left.\mathrm{NH}\right), 10.57\left(1 \mathrm{H}, \mathrm{bs}, \mathrm{D}_{2} \mathrm{O}\right.$ exchangeable, $\left.\mathrm{NH}\right), 7.86(2 \mathrm{H}, \mathrm{d}, J=8.0$ $\mathrm{Hz}, \mathrm{ArH}), 7.48-7.33(3 \mathrm{H}, \mathrm{m}, \mathrm{ArH}), 7.01\left(1 \mathrm{H}, \mathrm{s}, \mathrm{CH}\right.$-thiazole), $4.12\left(2 \mathrm{H}, \mathrm{q}, J=5.8 \mathrm{~Hz}, \mathrm{NHCH}_{2}\right)$, $3.69\left(3 \mathrm{H}, \mathrm{s}, \mathrm{OCH}_{3}\right), 2.83\left(2 \mathrm{H}, \mathrm{t}, J=5.8 \mathrm{~Hz}, \mathrm{CH}_{2}\right)$. Anal. Calcd. For $\mathrm{C}_{14} \mathrm{H}_{15} \mathrm{~N}_{3} \mathrm{O}_{2} \mathrm{~S}_{2}$ (321.4): C, 52.32; H, 4.70; N, 13.07; Found: C, 52.29; H, 4.64; N, 12.89.

Methyl 2-[3-(4-phenyl-thiazol-2-yl)-thioureido] propanoate (4c). From $\mathrm{L}-\mathrm{AlaOCH} \cdot \mathrm{HCl} 3 \mathrm{c}$ (0.7 g). Colorless crystals (0.85 g, $53 \%$ ); mp 170-171 ${ }^{\circ} \mathrm{C} .{ }^{1} \mathrm{H}$ NMR $\left(200 \mathrm{MHz}, \mathrm{CDCl}_{3}\right): \delta 11.68$ $\left(1 \mathrm{H}, \mathrm{bs}, \mathrm{D}_{2} \mathrm{O}\right.$ exchangeable, $\left.\mathrm{NH}\right), 10.71\left(1 \mathrm{H}, \mathrm{bs}, \mathrm{D}_{2} \mathrm{O}\right.$ exchangeable, $\left.\mathrm{NH}\right), 7.88(2 \mathrm{H}, \mathrm{d}, J=8.0$ $\mathrm{Hz}, \mathrm{ArH}), 7.47-7.30$ (3H, m, ArH), $7.02(1 \mathrm{H}, \mathrm{s}, \mathrm{CH}$-thiazole), 5.22-5.08 (1H, m, CH), $3.84(3 \mathrm{H}$, s, $\left.\mathrm{OCH}_{3}\right), 1.67\left(3 \mathrm{H}, \mathrm{d}, J=7.0 \mathrm{~Hz}, \mathrm{CH}_{3}\right)$. Anal. Calcd. For $\mathrm{C}_{14} \mathrm{H}_{15} \mathrm{~N}_{3} \mathrm{O}_{2} \mathrm{~S}_{2}$ (321.4): C, 52.32; $\mathrm{H}$, 4.70; N, 13.07; Found: C, 52.24; H, 4.68; N, 12.93.

Methyl 4-methyl-2-[3-(4-phenyl-thiazol-2-yl)-thioureido]-pentanoate (4d). From L$\mathrm{LeuOCH}_{3} \cdot \mathrm{HCl} 3 \mathrm{~d}(0.9 \mathrm{~g})$. Colorless crystals (1.05 g, $\left.57 \%\right)$; mp 152-153 ${ }^{\circ} \mathrm{C} .{ }^{1} \mathrm{H}$ NMR (200 $\left.\mathrm{MHz}_{\mathrm{CDCl}}\right): \delta 11.57\left(1 \mathrm{H}, \mathrm{bs}, \mathrm{D}_{2} \mathrm{O}\right.$ exchangeable, $\left.\mathrm{NH}\right), 10.93\left(1 \mathrm{H}, \mathrm{bs}, \mathrm{D}_{2} \mathrm{O}\right.$ exchangeable, $\left.\mathrm{NH}\right)$, $7.78(2 \mathrm{H}, \mathrm{d}, J=8.0 \mathrm{~Hz}, \mathrm{ArH}), 7.45-7.29(3 \mathrm{H}, \mathrm{m}, \mathrm{ArH}), 7.01(1 \mathrm{H}, \mathrm{s}, \mathrm{CH}-$ thiazole $), 5.12(1 \mathrm{H}, \mathrm{q}$, $J=7.2 \mathrm{~Hz}, \mathrm{CH}), 3.81\left(3 \mathrm{H}, \mathrm{s}, \mathrm{OCH}_{3}\right), 1.98-1.88\left(3 \mathrm{H}, \mathrm{m}, \mathrm{CH}_{2}, \mathrm{CH}\right), 1.03\left(3 \mathrm{H}, \mathrm{d}, J=5.8 \mathrm{~Hz}, \mathrm{CH}_{3}\right)$, $0.99\left(3 \mathrm{H}, \mathrm{d}, J=5.8 \mathrm{~Hz}, \mathrm{CH}_{3}\right)$. Anal. Calcd. For $\mathrm{C}_{17} \mathrm{H}_{21} \mathrm{~N}_{3} \mathrm{O}_{2} \mathrm{~S}_{2}(363.5)$ : C, 56.17; H, 5.82; N, 11.56; Found: C, 56.01; H, 5.66; N, 11.43 .

Methyl 4-[3-(4-phenyl-thiazol-2-yl)-thioureido] benzoate (6). ${ }^{29}$ From methyl 4-aminobenzoate 5 (0.75 g). Colorless crystals (1.4 g, $77 \%$ ); mp 201-202 ${ }^{\circ} \mathrm{C} .{ }^{1} \mathrm{H}$ NMR (200 MHz, $\left.\mathrm{CDCl}_{3}\right): \delta$ $13.25\left(1 \mathrm{H}, \mathrm{bs}, \mathrm{D}_{2} \mathrm{O}\right.$ exchangeable, $\left.\mathrm{NH}\right), 10.10\left(1 \mathrm{H}, \mathrm{bs}, \mathrm{D}_{2} \mathrm{O}\right.$ exchangeable, $\left.\mathrm{NH}\right), 8.15(2 \mathrm{H}, \mathrm{d}, J=$ $8.4 \mathrm{~Hz}, \mathrm{ArH}), 7.90(2 \mathrm{H}, \mathrm{d}, J=8.6 \mathrm{~Hz}, \mathrm{ArH}), 7.76(2 \mathrm{H}, \mathrm{d}, J=8.0 \mathrm{~Hz}, \mathrm{ArH}), 7.47-7.33(3 \mathrm{H}, \mathrm{m}$, $\mathrm{ArH}), 7.07$ (1H, s, CH-thiazole), $3.94\left(3 \mathrm{H}, \mathrm{s}, \mathrm{OCH}_{3}\right)$. Anal. Calcd. For $\mathrm{C}_{18} \mathrm{H}_{15} \mathrm{~N}_{3} \mathrm{O}_{2} \mathrm{~S}_{2}$ (369.5): C, 58.52; H, 4.09; N, 11.37; Found: C, 58.48; H, 4.03; N, 11.31.

1-Benzyl-3-(4-phenyl-thiazol-2-yl)-thiourea (7). ${ }^{29}$ From benzyl amine $(0.55 \mathrm{~mL})$. Colorless crystals $(1.1 \mathrm{~g}, 68 \%)$; mp 164-165 ${ }^{\circ} \mathrm{C} .{ }^{1} \mathrm{H}$ NMR $\left(200 \mathrm{MHz}, \mathrm{CDCl}_{3}\right): \delta 11.49\left(1 \mathrm{H}, \mathrm{bs}, \mathrm{D}_{2} \mathrm{O}\right.$ exchangeable, NH), 7.53-7.38 (5H, m, ArH), $7.31-7.22(5 \mathrm{H}, \mathrm{m}, \mathrm{ArH}), 6.95(1 \mathrm{H}, \mathrm{s}, \mathrm{CH}-$ thiazole), 6.38 (1H, bs, $\mathrm{D}_{2} \mathrm{O}$ exchangeable, $\left.\mathrm{NH}\right), 4.87\left(2 \mathrm{H}, \mathrm{d}, J=5.0 \mathrm{~Hz}, \mathrm{NHCH}_{2}\right)$. Anal. Calcd. For $\mathrm{C}_{17} \mathrm{H}_{15} \mathrm{~N}_{3} \mathrm{~S}_{2}$ (325.5): C, 62.74; H, 4.65; N, 12.91; Found: C, 62.68; H, 4.63; N, 12.88 .

\section{Derivatization reactions of $4 a, b$}

Ethyl [3-(4-phenyl-thiazol-2-yl)-thioureido] acetate (8). To a stirred solution of 4a (1.55 g, 5.0 $\mathrm{mmol})$ in absolute ethanol was added $\mathrm{KOH}(0.28 \mathrm{~g}, 5.0 \mathrm{mmol})$. The reaction mixture was refluxed for $4 \mathrm{~h}$, and then evaporated under reduced pressure. The residue was collected and crystallized from ethanol to give white crystals.

Colorless crystals (1.3 g, $82 \%)$; mp 198-199 ${ }^{\circ} \mathrm{C} .{ }^{1} \mathrm{H}$ NMR $\left(200 \mathrm{MHz}, \mathrm{CDCl}_{3}\right): \delta 11.48(1 \mathrm{H}$, bs, $\mathrm{D}_{2} \mathrm{O}$ exchangeable, $\left.\mathrm{NH}\right), 9.85\left(1 \mathrm{H}, \mathrm{bs}, \mathrm{D}_{2} \mathrm{O}\right.$ exchangeable, $\left.\mathrm{NH}\right), 7.87(2 \mathrm{H}, \mathrm{d}, J=8.0 \mathrm{~Hz}, \mathrm{ArH})$, 
7.48-7.32 (3H, m, ArH), 7.02 (1H, s, CH-thiazole), $4.61\left(2 \mathrm{H}, \mathrm{d}, J=4.6 \mathrm{~Hz}, \mathrm{NHCH}_{2}\right), 4.41(2 \mathrm{H}$, q, $\left.J=7.2 \mathrm{~Hz}, \mathrm{OCH}_{2}\right), 1.38\left(3 \mathrm{H}, \mathrm{t}, J=7.0 \mathrm{~Hz}, \mathrm{CH}_{3}\right)$. Anal. Calcd. For $\mathrm{C}_{14} \mathrm{H}_{15} \mathrm{~N}_{3} \mathrm{O}_{2} \mathrm{~S}_{2}(321.4)$ : C, 52.32; H, 4.70; N, 13.07; Found: C, 52.25; H, 4.66; N, 13.01 .

Reactions with alkyl halides. To a stirred mixture of 4a,b $(5.0 \mathrm{mmol})$ in absolute ethanol $(20$ $\mathrm{mL})$ and triethyl amine $(0.7 \mathrm{~mL}, 5.0 \mathrm{mmol})$ was added the appropriate alkyl halide [benzyl bromide, allyl bromide and ethyl chloroacetate] $(5.0 \mathrm{mmol})$. The reaction mixture was heated at $90{ }^{\circ} \mathrm{C}$ for $4 \mathrm{~h}$, then evaporated under reduced pressure. The residue was recrystallized from ethanol.

Methyl [2-allyl-3-(4-phenyl-thiazol-2-yl)-isothioureido]-acetate (9a). From Gly derivative 4a $(1.55 \mathrm{~g})$ and allyl bromide $(0.45 \mathrm{~mL})$. Colorless crystals $(0.85 \mathrm{~g}, 48 \%)$; mp ${ }^{\circ} \mathrm{C} .{ }^{1} \mathrm{H}$ NMR $(200$ $\left.\mathrm{MHz}_{\mathrm{CDCl}}\right): \delta 11.02\left(1 \mathrm{H}, \mathrm{bs}, \mathrm{D}_{2} \mathrm{O}\right.$ exchangeable, $\left.\mathrm{NH}\right), 7.93(2 \mathrm{H}, \mathrm{d}, J=8.0 \mathrm{~Hz}, \mathrm{ArH}), 7.45-$ $7.28(3 \mathrm{H}, \mathrm{m}, \mathrm{ArH}), 7.03(1 \mathrm{H}, \mathrm{s}, \mathrm{CH}$-thiazole $), 6.18-5.83\left(1 \mathrm{H}, \mathrm{m}, \mathrm{CH}=\mathrm{CH}_{2}\right), 5.31(1 \mathrm{H}, \mathrm{d}, J=17.0$ $\left.\mathrm{Hz}, \mathrm{CH}=\mathrm{CH}_{2}\right), 5.15\left(1 \mathrm{H}, \mathrm{d}, J=9.8 \mathrm{~Hz}, \mathrm{CH}=\mathrm{CH}_{2}\right), 4.22\left(2 \mathrm{H}, \mathrm{d}, J=4.6 \mathrm{~Hz}, \mathrm{NHCH}_{2}\right), 3.85-3.78$ $\left(5 \mathrm{H}, \mathrm{m}, \mathrm{SCH}_{2}, \mathrm{OCH}_{3}\right)$. Anal. Calcd. For $\mathrm{C}_{16} \mathrm{H}_{17} \mathrm{~N}_{3} \mathrm{O}_{2} \mathrm{~S}_{2}$ (347.5): C, 55.31; H, 4.93; N, 12.09; Found: C, 55.26; H, 4.84; N, 12.01 .

Methyl 3-[2-allyl-3-(4-phenyl-thiazol-2-yl)-isothioureido]-propanoate (9b). From $\beta$-Ala derivative $4 \mathbf{b}(1.6 \mathrm{~g})$ and allyl bromide $(0.45 \mathrm{~mL})$. Colorless crystals $(1.1 \mathrm{~g}, 60 \%)$; $\mathrm{mp} 75-76{ }^{\circ} \mathrm{C}$. ${ }^{1} \mathrm{H}$ NMR (200 MHz, $\left.\mathrm{CDCl}_{3}\right): \delta 10.68\left(1 \mathrm{H}, \mathrm{bs}, \mathrm{D}_{2} \mathrm{O}\right.$ exchangeable, $\left.\mathrm{NH}\right), 7.83(2 \mathrm{H}, \mathrm{d}, J=7.0 \mathrm{~Hz}$, ArH), 7.45-7.27 (3H, m, ArH), $7.00\left(1 \mathrm{H}, \mathrm{s}, \mathrm{CH}\right.$-thiazole), 6.06-5.86 (1H, m, $\left.\mathrm{CH}=\mathrm{CH}_{2}\right), 5.31$ $\left(1 \mathrm{H}, \mathrm{d}, J=16.8 \mathrm{~Hz}, \mathrm{CH}=\mathrm{CH}_{2}\right), 5.15\left(1 \mathrm{H}, \mathrm{d}, J=10.8 \mathrm{~Hz}, \mathrm{CH}=\mathrm{CH}_{2}\right), 3.82(2 \mathrm{H}, \mathrm{d}, J=7.0 \mathrm{~Hz}$, $\left.\mathrm{SCH}_{2}\right), 3.77-3.70\left(5 \mathrm{H}, \mathrm{m}, \mathrm{NCH}_{2}, \mathrm{OCH}_{3}\right), 2.72\left(2 \mathrm{H}, \mathrm{t}, J=6.0 \mathrm{~Hz}, \mathrm{CH}_{2}\right)$. Anal. Calcd. For $\mathrm{C}_{17} \mathrm{H}_{19} \mathrm{~N}_{3} \mathrm{O}_{2} \mathrm{~S}_{2}$ (361.5): C, 56.48; H, 5.30; N, 11.62; Found: C, 56.35; H, 5.28; N, 11.46.

Methyl [2-benzyl-3-(4-phenyl-thiazol-2-yl)-isothioureido]-acetate (10a). From Gly derivative 4a $(1.55 \mathrm{~g})$ and benzyl bromide $(0.6 \mathrm{~mL})$. Colorless crystals $(1.55 \mathrm{~g}, 78 \%) ; \mathrm{mp} 102-103{ }^{\circ} \mathrm{C} .{ }^{1} \mathrm{H}$ NMR (200 MHz, CDCl $): \delta 11.04\left(1 \mathrm{H}, \mathrm{bs}, \mathrm{D}_{2} \mathrm{O}\right.$ exchangeable, $\left.\mathrm{NH}\right), 7.97-7.88(2 \mathrm{H}, \mathrm{m}, \mathrm{ArH})$, 7.46-7.27 (8H, m, ArH), $7.05\left(1 \mathrm{H}, \mathrm{s}, \mathrm{CH}\right.$-thiazole), $4.42\left(2 \mathrm{H}, \mathrm{s}, \mathrm{SCH}_{2}\right), 4.19(2 \mathrm{H}, \mathrm{d}, J=5.0 \mathrm{~Hz}$, $\mathrm{NCH}_{2}$ ), $3.85\left(3 \mathrm{H}, \mathrm{s}, \mathrm{OCH}_{3}\right)$. Anal. Calcd. For $\mathrm{C}_{20} \mathrm{H}_{19} \mathrm{~N}_{3} \mathrm{O}_{2} \mathrm{~S}_{2}(397.5)$ : C, 60.43; H, 4.82; N, 10.57; Found: C, 60.38; H, 4.74; N, 10.43;

Methyl 3-[2-benzyl-3-(4-phenyl-thiazol-2-yl)-isothioureido]-propanoate (10b). From $\beta$-Ala derivative $4 \mathbf{b}(1.6 \mathrm{~g})$ and benzyl bromide $(0.6 \mathrm{~mL})$. Colorless crystals $(1.75 \mathrm{~g}, 85 \%)$; mp 88-89 ${ }^{\circ} \mathrm{C} .{ }^{1} \mathrm{H}$ NMR $\left(200 \mathrm{MHz}, \mathrm{CDCl}_{3}\right): \delta 10.68\left(1 \mathrm{H}, \mathrm{bs}, \mathrm{D}_{2} \mathrm{O}\right.$ exchangeable, $\left.\mathrm{NH}\right), 7.84(2 \mathrm{H}, \mathrm{d}, J=7.0$ $\mathrm{Hz}, \mathrm{ArH}), 7.46-7.27$ (8H, m, ArH), $7.01(1 \mathrm{H}, \mathrm{s}, \mathrm{CH}$-thiazole), 4.41 (2H, s, SCH$), 3.74-3.65$ $\left(5 \mathrm{H}, \mathrm{m}, \mathrm{NCH}_{2}, \mathrm{OCH}_{3}\right), 2.68\left(2 \mathrm{H}, \mathrm{t}, J=6.4 \mathrm{~Hz}, \mathrm{CH}_{2}\right)$. Anal. Calcd. For $\mathrm{C}_{21} \mathrm{H}_{21} \mathrm{~N}_{3} \mathrm{O}_{2} \mathrm{~S}_{2}$ (411.5): C, 61.29; H, 5.14; N, 10.21; Found: C, 61.15; H, 5.07; N, 10.13.

Methyl [4-Oxo-2-(4-phenyl-thiazol-2-ylimino)-thiazolidin-3-yl]-acetate (11a). From Gly derivative $4 \mathrm{a}(1.55 \mathrm{~g})$ and ethyl chloroacetate $(0.55 \mathrm{~mL})$. Colorless crystals $(1.05 \mathrm{~g}, 61 \%)$; $\mathrm{mp}$ 182-183 ${ }^{\circ} \mathrm{C} .{ }^{1} \mathrm{H}$ NMR $\left(200 \mathrm{MHz}, \mathrm{CDCl}_{3}\right): \delta$ 7.92-7.86 (2H, m, ArH), 7.46-7.29 (4H, m, 3ArH, $\mathrm{CH}$-thiazole $), 4.64\left(2 \mathrm{H}, \mathrm{s}, \mathrm{NCH}_{2}\right), 3.93\left(2 \mathrm{H}, \mathrm{s}, \mathrm{SCH}_{2}\right), 3.78\left(3 \mathrm{H}, \mathrm{s}, \mathrm{OCH}_{3}\right)$. Anal. Calcd. For $\mathrm{C}_{15} \mathrm{H}_{13} \mathrm{~N}_{3} \mathrm{O}_{3} \mathrm{~S}_{2}$ (347.4): C, 51.86; H, 3.77; N, 12.10; Found: C, 51.81; H, 3.64; N, 12.96 . 
Methyl 3-[4-Oxo-2-(4-phenyl-thiazol-2-ylimino)-thiazolidin-3-yl]-propanoate (11b). From $\beta$ Ala derivative $4 \mathbf{b}(1.6 \mathrm{~g})$ and ethyl chloroacetate $(0.55 \mathrm{~mL})$. Colorless crystals $(1.55 \mathrm{~g}, 87 \%)$; mp 165-166 ${ }^{\circ} \mathrm{C} .{ }^{1} \mathrm{H}$ NMR (200 MHz, $\left.\mathrm{CDCl}_{3}\right): \delta 7.90(2 \mathrm{H}, \mathrm{d}, J=7.8 \mathrm{~Hz}, \mathrm{ArH}), 7.46-7.32(4 \mathrm{H}$, m, 3ArH, CH-thiazole), $4.19\left(2 \mathrm{H}, \mathrm{t}, J=7.4 \mathrm{~Hz}, \mathrm{NCH}_{2}\right), 3.8\left(2 \mathrm{H}, \mathrm{s}, \mathrm{SCH}_{2}\right), 3.69\left(3 \mathrm{H}, \mathrm{s}, \mathrm{OCH}_{3}\right)$, $2.74\left(2 \mathrm{H}, \mathrm{t}, J=7.4 \mathrm{~Hz}, \mathrm{CH}_{2}\right)$. Anal. Calcd. For $\mathrm{C}_{16} \mathrm{H}_{15} \mathrm{~N}_{3} \mathrm{O}_{3} \mathrm{~S}_{2}(361.4)$ : C, 53.17; H, 4.18; N, 11.63; Found: C, 53.03; H, 4.16; N, 11.54.

\section{Reactions with phenacyl bromides}

To a stirred mixture of $4 a(1.55 \mathrm{~g}, 5.0 \mathrm{mmol})$ in DMSO $(10 \mathrm{~mL})$ and triethyl amine $(0.7 \mathrm{~mL}, 5.0$ mmol) was added the appropriate phenacyl bromide $12(5.0 \mathrm{mmol})$. The reaction mixture was left at room temperature over night, poured over water. The resultant solid was filtered off and recrystallized from ethanol.

Methyl [4-hydroxy-4-phenyl-2-(4-phenyl-thiazol-2-ylimino)-thiazolidin-3-yl]-acetate (13a). From Gly derivative 4a (1.55 g) and phenacyl bromide 12a (1.0 g). Yellowish orange crystals $(1.3 \mathrm{~g}, 62 \%) ; \mathrm{mp} 45-47{ }^{\circ} \mathrm{C} .{ }^{1} \mathrm{H}$ NMR $\left(200 \mathrm{MHz}, \mathrm{CDCl}_{3}\right): \delta 7.92(2 \mathrm{H}, \mathrm{d}, J=7.0 \mathrm{~Hz}, \mathrm{ArH}), 7.61$ $(2 \mathrm{H}, \mathrm{d}, J=7.8 \mathrm{~Hz}, \mathrm{ArH}), 7.47-7.28(6 \mathrm{H}, \mathrm{m}, \mathrm{ArH}), 7.11(1 \mathrm{H}, \mathrm{s}, \mathrm{CH}$-thiazole $), 4.85\left(1 \mathrm{H}, \mathrm{bs}, \mathrm{D}_{2} \mathrm{O}\right.$ exchangeable, $\mathrm{OH}), 4.46\left(1 \mathrm{H}, \mathrm{d}, J=17.2 \mathrm{~Hz}, \mathrm{NCH}_{\mathrm{A}}\right), 3.77-3.66\left(4 \mathrm{H}, \mathrm{m}, \mathrm{OCH}_{3}, \mathrm{NCH}_{\mathrm{B}}\right), 3.60$ $\left(1 \mathrm{H}, \mathrm{d}, J=11.8 \mathrm{~Hz}, \mathrm{SCH}_{\mathrm{A}}\right), 3.52\left(1 \mathrm{H}, \mathrm{d}, J=11.8 \mathrm{~Hz}, \mathrm{SCH}_{\mathrm{B}}\right)$. Anal. Calcd. For $\mathrm{C}_{21} \mathrm{H}_{19} \mathrm{~N}_{3} \mathrm{O}_{3} \mathrm{~S}_{2}$ (425.5): C, 59.27; H, 4.50; N, 9.87; Found: C, 59.12; H, 4.34; N, 9.67.

Methyl [4-Hydroxy-2-(4-phenyl-thiazol-2-ylimino)-4-p-tolyl-thiazolidin-3-yl]-acetate (13b). From Gly derivative 4a (1.55 g) and 4-methyl phenacyl bromide 12b (1.05 g). Brownish crystals (0.95 g, $43 \%$ ); mp 40-41 ${ }^{\circ} \mathrm{C} .{ }^{1} \mathrm{H}$ NMR $\left(200 \mathrm{MHz}, \mathrm{CDCl}_{3}\right): \delta 7.90(2 \mathrm{H}, \mathrm{d}, J=7.2 \mathrm{~Hz}, \mathrm{ArH})$, 7.48-7.17 (7H, m, ArH), 7.09 (1H, s, CH-thiazole), 4.78 (1H, bs, $\mathrm{D}_{2} \mathrm{O}$ exchangeable, $\left.\mathrm{OH}\right), 4.40$ $\left(1 \mathrm{H}, \mathrm{d}, J=17.2 \mathrm{~Hz}, \mathrm{NCH}_{\mathrm{A}}\right), 3.70\left(3 \mathrm{H}, \mathrm{s}, \mathrm{OCH}_{3}\right), 3.63\left(1 \mathrm{H}, \mathrm{d}, J=17.2 \mathrm{~Hz}, \mathrm{NCH}_{\mathrm{B}}\right), 3.57(1 \mathrm{H}, \mathrm{d}$, $\left.J=11.8 \mathrm{~Hz}, \mathrm{SCH}_{\mathrm{A}}\right), 3.48\left(1 \mathrm{H}, \mathrm{d}, J=11.8 \mathrm{~Hz}, \mathrm{SCH}_{\mathrm{B}}\right), 2.34\left(3 \mathrm{H}, \mathrm{s}, \mathrm{CH}_{3}\right)$. Anal. Calcd. For $\mathrm{C}_{22} \mathrm{H}_{21} \mathrm{~N}_{3} \mathrm{O}_{3} \mathrm{~S}_{2}$ (439.6): C, 60.11; H, 4.82; N, 9.56; Found: C, 60.04; H, 4.76; N, 9.51.

Methyl [4-hydroxy-4-(4-methoxy-phenyl)-2-(4-phenyl-thiazol-2-ylimino)-thiazolidin-3-yl]acetate (13c). From Gly derivative 4a (1.55 g) and 4-methoxy phenacyl bromide 12c (1.15 g). Colorless crystals $(1.3 \mathrm{~g}, 57 \%)$; mp 50-51 ${ }^{\circ} \mathrm{C} .{ }^{1} \mathrm{H}$ NMR $\left(200 \mathrm{MHz}, \mathrm{CDCl}_{3}\right): \delta 7.92(2 \mathrm{H}, \mathrm{d}, J=$ $7.2 \mathrm{~Hz}, \mathrm{ArH}), 7.52(2 \mathrm{H}, \mathrm{d}, J=8.6 \mathrm{~Hz}, \mathrm{ArH}), 7.43-7.27$ (3H, m, ArH), 7.11 (1H, s, CH-thiazole), $6.92(2 \mathrm{H}, \mathrm{d}, J=8.6 \mathrm{~Hz}, \mathrm{ArH}), 4.80\left(1 \mathrm{H}, \mathrm{bs}, \mathrm{D}_{2} \mathrm{O}\right.$ exchangeable, $\left.\mathrm{OH}\right), 4.44(1 \mathrm{H}, \mathrm{d}, J=17.0 \mathrm{~Hz}$, $\left.\mathrm{NCH}_{\mathrm{A}}\right), 3.81\left(3 \mathrm{H}, \mathrm{s}, \mathrm{OCH}_{3}\right), 3.72\left(3 \mathrm{H}, \mathrm{s}, \mathrm{OCH}_{3}\right), 3.63\left(1 \mathrm{H}, \mathrm{d}, J=17.0 \mathrm{~Hz}, \mathrm{NCH}_{\mathrm{B}}\right), 3.58(1 \mathrm{H}, \mathrm{d}$, $\left.J=12.0 \mathrm{~Hz}, \mathrm{SCH}_{\mathrm{A}}\right), 3.49\left(1 \mathrm{H}, \mathrm{d}, J=12.0 \mathrm{~Hz}, \mathrm{SCH}_{\mathrm{B}}\right), 2.34\left(3 \mathrm{H}, \mathrm{s}, \mathrm{CH}_{3}\right)$. Anal. Calcd. For $\mathrm{C}_{22} \mathrm{H}_{21} \mathrm{~N}_{3} \mathrm{O}_{4} \mathrm{~S}_{2}$ (455.6): C, 58.00; H, 4.65; N, 9.22; Found: C, 57.89; H, 4.54; N, 9.13.

\section{References}

1. Morris, K. Lancet 1997, 350, 1683.

2. El Safadi, Y.; Vivet-Boudou, V.; Marquet, R. Appl. Microbiol. Biotechnol. 2007, 75, 723. 
3. Patick, A. K.; Potts, K. E. Clin. Microbiol. Rev. 1998, 11, 614.

4. De Clercq, E. Med. Res. Rev. 1996, 16, 125.

5. Romero, D. L.; Olmsted, R. A.; Poel, T. J.; Morge, R. A.; Biles, C.; Keiser, B. J.; Kopta, L. A.; Frils, J. M.; Hosley, J. D.; Stefanski, K. J.; Wishka, D. G.; Evans, D. B.; Morris, J.; Stehle, R. G.; Sharma, S. K.; Yagi, Y.; Voorman, R. L.; Adams, W. J.; Tarpley, W. G. J. Med. Chem. 1996, 39, 3769.

6. Ahgren, C.; Backro, K.; Bell, F. W.; Cantrell, A. S.; Clemens, M.; Colacino, J.; Deeter, M. J. B.; Engelhardt, J. A.; Jaskunas, S. R.; Johansson, N. G.; Jordan, C. L.; Kasher, J. S.; Kinnick, M. D.; Lind, P.; Lopez, C.; Morin, J. M.; Muesing, M. A.; Noreen, R.; Oberg, B.; Paget, C. J.; Palkowitz, J. A.; Parrish, C.; Pranc, P.; Rippy, M. K.; Rydergard, C.; Sahlberg, C.; Swanson, S.; Ternansky, R.; Unge, J.; Vasileff, T. R. T.; Vrang, L.; West, S. J.; Zhang, H.; Zhou, X. X. Antimicrob. Agents Chemother. 1995, 39, 1329.

7. Heinisch, G.; Matuszczak, B.; Pachler, S.; Rakowitz, D. Antivir. Chem. Chemother. 1997, 8, 443.

8. Ren, J.; Diprose, J.; Warren, J.; Esnouf, R. M.; Bird, L. E.; Ikemizu, S.; Slater, M.; Milton, J.; Balzarini, J.; Stuart, D. I; Stammers, D. K. J. Biol. Chem. 2000, 275, 5633.

9. Ahgren, C.; Backro, K.; Bell, F. W.; Cantrell, S.; Clemens, M.; Colacino, J. M.; Deeter, M. J. B.; Engelhardt, J. A.; Hogberg, M.; Jaskunas, S. R. Antimicrob. Agents Chemother. 1995, 39, 1329.

10. Uckun, F. M.; Venkatachalam, T. K. United States Patent 2005, 6960606.

11. Fathalla, W.; Pazdera, P. ARKIVOC 2007, (i), 236.

12. Fathalla, W.; Ali, I. A. I. Heteroatom Chem. 2007, 18, 637.

13. Fathalla, W.; El Rayes, S. M.; Ali, I. A. I. ARKIVOC 2007, (xvi), 173.

14. El Rayes, S. M.; Ali, I. A. I.; Fathalla, W. ARKIVOC 2008, (xi), 86.

15. Abdelhamid, A. O.; Abdel-Riheem, N. A.; Hassan, N. M. Heteroatom Chem. 2007, 11, 213.

16. Cruz, A.; Padilla-Martínez, I. I.; García-Báez, E. V.; Juárez, M. J. ARKIVOC 2008, (v), 200.

17. Venkatachalam, T. K.; Sudbeck, E.; Uckun, F. M. J. Mol. Struc. 2005, 751, 41.

18. Venkatachalam, T. K.; Sudbeck, E.; Uckun, F. M. J. Mol. Struc. 2004, 687, 45.

19. Venkatachalam, T. K.; Uckun, F. M. Synth. Commun. 2006, 36, 2103.

20. Tsogoeva, S. B.; Hateley, M. J.; Yalalov, D. A.; Meindl, K.; Weckbeckerb, C; Huthmacherb, K. Bioorg. Med. Chem. 2005, 13, 5680.

21. Plass, M.; Wawer, I.; Piekarska-Bartoszewicz, B.; Temeriusz, A. J. Phys. Org. Chem. 1998, $10,747$.

22. Wuckelt, J.; Döring, M.; Langer, P.; Görls, H.; Beckert, R. Tetrahedron Lett. 1997, 38, 5269.

23. Bilek, P.; Slouka, J. Heterocycl. Commun. 2002, 8, 123.

24. Fathalla, W.; Čajan, M.; Pazdera, P. Molecules 2001, 6, 557.

25. Fathalla, W.; Čajan, M.; Pazdera, P. Molecules 2000, 6, 1210.

26. Fathalla, W.; Čajan, M.; Pazdera, P. J. Heterocycl. Chem. 2002, 39, 1145. 
27. Barreca, M. L.; Chimirri, A.; De Luca, L.; Monforte, A-M.; Monforte, P.; Rao, A.; Zappalà, M.; Balzarini, J.; De Clercq, E.; Pannecouque, C.; Witvrouw, M. Bioorg. Med. Chem. Lett. 2001, 11, 1793.

28. Liu, H-L.; Zongcheng Li, Z.; Anthonsen, T. Molecules 2000, 5, 1055.

29. Fathalla, W.; Čajan, M.; Marek, J. Pazdera, P. Molecules 2001, 6, 588. 